\title{
DESARROLLO DE UN SISTEMA PARA PROGRAMAR RECURSOS Y OPERACIONES EN LA FABRICACIÓN DE CALENTADORES ELÉCTRICOS BAJO PEDIDO
}

\author{
Ana Castillo K. ${ }^{1} \quad$ David Muñoz N. $^{2}$ \\ Recibido el 9 de marzo de 2004, aceptado el 12 de septiembre de 2004
}

\begin{abstract}
RESUMEN
Una empresa mexicana de calentadores de agua eléctricos desea estimar el menor tiempo de producción de los calentadores que se hacen bajo pedido. Con el objetivo de minimizar el tiempo de entrega de sus órdenes, se deben programar apropiadamente los recursos y las actividades productivas. En este trabajo se presenta el desarrollo de un sistema de apoyo a la toma de decisiones para estimar el tiempo mínimo de producción en una orden de calentadores eléctricos. El sistema se desarrolló en MS Excel ${ }^{\circledR}$ con el uso de macros y MS Visual Basic ${ }^{\circledR}$. A partir de los datos de la orden de producción, el sistema genera la red de actividades necesarias para producir la orden, y con base en algoritmos para resolver el problema de programación de actividades con recursos limitados, se propone una programación de recursos y actividades que trata de minimizar el tiempo de producción. Debido a que el número de actividades que se requieren para satisfacer una orden típica de producción es grande, el sistema utiliza heurísticas para resolver el problema de programación. En particular, se propone una heurística que utiliza tiempos de ocio para tratar de minimizar el tiempo de producción, y que luego de probarse demostró, en muchos casos, un mejor desempeño que la heurística de menor holgura.
\end{abstract}

Palabras claves: Programación de actividades, recursos limitados, programación de la producción, métodos heurísticos, tiempo de respuesta.

\begin{abstract}
A Mexican company produces water heaters under a make to order production strategy, and wishes to estimate its response time to deliver orders from its clients. In order to minimize the response time for a given order, the plant needs to schedule resources and operations accordingly. In this paper we present a decision support system that was developed to assist the plant manager in the estimation of the response time to deliver the heaters specified in a given order. The system was developed in MS Excel ${ }^{\circledR}$, and uses $M S V B A^{\circledR}$ macros. After an order is entered in the system, a network representing the sequence of required operations is constructed, and algorithms for resource-constrained project scheduling (RCPS) are applied in order to suggest an appropriate operations schedule and resource allocation. Since the number of operations required to deliver a given order of heaters is typically large, the algorithms used for RCPS are heuristics. A particular heuristic procedure that was designed and implemented in this project inserts resource idle times trying to reduce the response time for an order, and was shown to perform better than the minimum slack heuristic in many cases.
\end{abstract}

Keywords: Resource-constrained project scheduling, production planning, sequencing, heuristics, response time.

\section{INTRODUCCIÓN}

Una empresa mexicana fabrica calentadores eléctricos de agua desde 1949 con un nivel relativamente bajo de automatización. Algunos de los pedidos corresponden a modelos estandarizados, que se abastecen inmediatamente con el inventario disponible, aunque la empresa a menudo acepta pedidos de calentadores con características especiales solicitadas por el cliente. Desde hace cinco años el negocio ha venido enfrentado la competencia de otras empresas que producen modelos estandarizados de calentadores eléctricos, utilizando procesos de producción en serie y altamente automatizados, logrando bajar considerablemente costos, y por lo tanto precios.

\footnotetext{
${ }^{1}$ Calentadores Keller, México D.F., México. anacastillok@yahoo.com

${ }^{2}$ Departamento de Ingeniería Industrial y de Operaciones, Instituto Tecnológico Autónomo de México, México D.F., México. davidm@itam.mx
} 
Debido a que la empresa se ha caracterizado por satisfacer las características de diseño que piden sus clientes, mercado en el cual hay un menor número de empresas competidoras, se ha tomado la decisión de enfocarse principalmente a la fabricación de productos especiales, es decir, a la producción de calentadores no estandarizados bajo pedido. El enfoque de la empresa en el mercado de calentadores especiales, sin embargo, requiere del desarrollo de capacidades adecuadas para satisfacer los requerimientos de sus clientes, en particular, se debe tratar de reducir el tiempo necesario para satisfacer el pedido de una orden de compra determinada (tiempo de ciclo), cuyo componente fundamental es el tiempo de fabricación de los calentadores solicitados en la orden, ya que no se tienen inventarios para abastecer este mercado.

Al momento de iniciar este proyecto, no se tenía una idea clara de los tiempos y los costos de fabricación, debido fundamentalmente a que el proceso de manufactura no estaba estandarizado, y a que no se disponía de registros de los tiempos de las operaciones necesarias para producir los diferentes modelos. Esta falta de información era la principal causa de que en ciertas ocasiones la entrega de los pedidos de calentadores especiales se haya hecho después de la fecha de entrega establecida, lo que podría evitarse si la empresa pudiera programar adecuadamente sus operaciones productivas, tratando de reducir al mínimo sus tiempos de ciclo, y permitiendo también una mejor estimación de sus fechas de entrega. Por estas razones es que se decidió desarrollar un sistema para estimar el menor tiempo de fabricación (tiempo de ciclo) de una orden de calentadores especiales, que además de permitir una mejor estimación de la fecha de entrega, permitiría a la empresa obtener otras ventajas competitivas derivadas de la reducción de sus tiempos de ciclo (véase por ejemplo, [1]).

\section{PROCESO DE PRODUCCIÓN}

Para desarrollar el sistema fue necesario estudiar al producto así como su proceso productivo. La empresa fabrica calentadores eléctricos en diferentes tamaños, desde 15 hasta 1000 litros de capacidad, y aunque las dimensiones y los requerimientos de energía cambian conforme al tamaño, todos utilizan los mismos elementos para operar, excepto los calentadores de 1000 litros, por lo que se hizo una lista de los materiales que se requieren para la fabricación de los calentadores de 15 a 700 litros y otra para los calentadores de 1000 litros. Asimismo, se construyó un árbol de materiales (del término inglés Bill of materials, veáse [4]) para los calentadores de 15 a 700 litros y para los calentadores de 1000 litros, en donde se muestran los materiales requeridos para la manufactura de cada uno de los componentes de un calentador [2].

A continuación se registró el inventario de máquinas y de operadores disponibles, la disposición de la planta, y un análisis de qué tipo de actividades puede realizar cada máquina. Posteriormente, se estudió el proceso de producción de los calentadores y debido a la gran variedad de modelos de calentadores se optó por agrupar los modelos cuyos procesos y tiempos de producción fueran similares, teniendo así cuatro grupos diferentes de calentadores (ver Tabla 1 y Tabla 2):

a) 15 a 80 litros: son calentadores pequeños para uso residencial o pequeños comercios estilo cafeterías, salones de belleza etc.

b) 100 a 240 litros: estos calentadores generalmente se utilizan en los comercios o en residencias de gran tamaño.

c) 300 a 700 litros: principalmente son calentadores de uso industrial.

d) 1000 litros: son grandes calentadores industriales de producción esporádica.

Para cada grupo de calentadores se diseñó un diagrama del proceso de operaciones en donde se muestran todas las operaciones o actividades necesarias para formar cada parte del calentador y las relaciones de precedencia que hay entre ellas. Para la producción de un calentador hay en promedio 45 actividades a realizar y una orden típica de calentadores, que consta de al menos 3 diferentes tipos de calentadores, se compone de 140 actividades o más. Finalmente, a partir del diagrama de proceso de operaciones, se configuró una lista de operaciones. En ésta se encuentra toda la información suficiente y necesaria para la ejecución del sistema, es decir, las características de cada actividad: las precedencias, la maquinaria y el personal necesarios para realizar la actividad así como el tiempo total para llevarla a cabo.

Tabla 1. Agrupaciones de los calentadores eléctricos.

\begin{tabular}{|c|c|c|c|}
\hline $\begin{array}{c}\text { CE-15 a 80 } \\
\text { lt. }\end{array}$ & $\begin{array}{c}\text { CE-100 a } \\
\text { 240 lt. }\end{array}$ & $\begin{array}{c}\text { CE-300 a } \\
\mathbf{7 0 0} \text { lt. }\end{array}$ & CE-1000 lt. \\
\hline CE-15 & CE-100 & CE-300 & CE-1000 \\
\hline CE-30 & CE-120 & CE-350 & \\
\hline CE-40 & CE-180 & CE-400 & \\
\hline CE-60 & CE-200 & CE-500 & \\
\hline CE-80 & CE-240 & CE-700 & \\
\hline
\end{tabular}


Tabla 2. Porcentaje de demanda e ingreso por grupo de calentadores (2000).

\begin{tabular}{|c|c|c|}
\hline $\begin{array}{c}\text { Tipo de } \\
\text { calentador }\end{array}$ & $\begin{array}{c}\text { \% de la } \\
\text { demanda total }\end{array}$ & $\begin{array}{c}\text { \% de ingreso } \\
\text { total }\end{array}$ \\
\hline CE-15 a 80 lt. & $80 \%$ & $52 \%$ \\
\hline CE-100 a 240 lt. & $16 \%$ & $25 \%$ \\
\hline CE-300 a 700 lt. & $4 \%$ & $23 \%$ \\
\hline CE-1000 lt. & $0 \%$ & $0 \%$ \\
\hline
\end{tabular}

Para calcular el tiempo total de cada actividad se tomaron en cuenta diferentes tiempos, los que se presentan en la Tabla 3. Para la estimación de estos tiempos se tomó el promedio de diez mediciones de cada operación en el momento de la fabricación de los calentadores.

Con la finalidad de reducir los tiempos por apertura de procesos (setup), se producen simultáneamente (por lote) todas las unidades del mismo tipo de calentador solicitadas en la orden de producción, razón por la cual el tiempo total de cada actividad se obtuvo de la siguiente manera:

$\mathrm{T}$. total $=\mathrm{T}$. total fijo $+(\mathrm{T}$. total variable $*$ Número de productos deseado)

Se consideraron como tiempos fijos los tiempos que se definen en la Tabla 3 con los nombres Setup2, Setup3 y Externo. Estos tiempos no dependen del volumen de la orden. Los tiempos variables son los que dependen del número de calentadores que se ordenen, y son los que corresponden a: Setup, Trazo, Setup1, Setup4 y Operación (ver Tabla 3).

Tabla 3. Nombre y descripción de los diferentes tiempos utilizados.

\begin{tabular}{|l|l|}
\hline Nombre & \multicolumn{1}{|c|}{ Descripción } \\
\hline Setup & Transporte del material a la zona de trazo \\
\hline Trazo & Trazo de materiales \\
\hline Setup1 & $\begin{array}{l}\text { Transporte del material a la zona donde se } \\
\text { realiza la operación }\end{array}$ \\
\hline Setup2 & $\begin{array}{l}\text { Cambio de herramientas y/o colocación de } \\
\text { topes en la máquina }\end{array}$ \\
\hline Setup3 & $\begin{array}{l}\text { Conexión y preparación de la máquina para } \\
\text { realizar una operación }\end{array}$ \\
\hline Setup4 & Colocación de una pieza en la máquina \\
\hline Externo & Operación fuera de la fábrica \\
\hline Operación & Operación \\
\hline
\end{tabular}

\section{CARACTERÍSTICAS DEL SISTEMA}

El sistema se desarrolló en un libro de cálculo de Excel, en una de cuyas hojas se captura el número de calentadores de cada tipo que se solicitan en la orden, y para cada tipo de calentador se mantiene una hoja con información sobre su red de actividades (lista de operaciones), fundamentalmente los tiempos estándares de cada actividad, las relaciones de precedencia, y los recursos (humanos y de capital) que se utilizan en cada actividad. Con base en esta información, el sistema construye la red de actividades (para mayor detalle, véase [2]) necesaria para completar la producción de los calentadores de la orden, y asumiendo que cuando se comienza a trabajar en una orden de producción, ésta no debe ser interrumpida hasta terminarse, el sistema propone la programación de actividades en la red, tratando de minimizar el tiempo de ciclo, y considerando las restricciones de recursos disponibles.

Si no existieran restricciones de recursos, el tiempo de ciclo mínimo para una orden podría obtenerse a partir de los tiempos que requieren cada una de las operaciones de la red de actividades, utilizando el método de la ruta crítica (véase por ejemplo, [1]). Sin embargo, debido a que no se tienen recursos (humanos y de capital) ilimitados para ejecutar las actividades, se debe buscar una asignación de recursos y una secuenciación de actividades tratando de minimizar el tiempo de ciclo, problema que se conoce en la literatura inglesa con el nombre de resource-constrained project scheduling (RCPS) (véase por ejemplo, [5]).

Para atacar el problema RCPS se han propuesto metodologías que se clasifican en: (a) métodos exactos, y (b) reglas heurísticas. Los métodos exactos permiten encontrar la duración mínima del proyecto, es decir, la solución exacta del problema, pero tienen limitaciones para resolver problemas con un gran número de actividades o de recursos en un tiempo razonable de cómputo. Se han propuesto algoritmos basados en el principio de ramificación y acotamiento (branch and bound), especialmente diseñados para este problema [6], [7]. Sin embargo, este método no encuentra el óptimo a partir de un cierto tamaño (más de 12 recursos o más de 40 actividades). Considerando que la red de actividades que genera una orden típica de calentadores es relativamente grande (a menudo más de 140 actividades y 13 recursos) se decidió implementar reglas heurísticas buscando acercarse lo más posible al tiempo de ciclo mínimo. A continuación se presenta una descripción de las heurísticas desarrolladas en el sistema. 


\section{Estructura Clásica de un Método Heurístico}

Los métodos basados en reglas heurísticas a menudo se aplican de acuerdo con el procedimiento que se indica a continuación (véase por ejemplo, [5]).

Se inicializan todas las actividades como no programadas, y se determinan las actividades candidatas. En cualquier momento se dice que una actividad es candidata si tiene todas sus actividades precedentes completas, y existen los recursos necesarios para su ejecución. Luego de seleccionar las actividades candidatas, éstas se van programando de acuerdo con el criterio de prioridad propio de la heurística, teniendo en cuenta que al programar una actividad disminuye la disponibilidad de recursos, y se deben determinar nuevamente las actividades candidatas. Cuando ya no existen actividades candidatas, se avanza el reloj hasta la finalización de la actividad más próxima, donde se liberan recursos, y se repite el procedimiento, es decir, se determinan las actividades candidatas y se programan actividades. El tiempo de terminación o tiempo de ciclo del proyecto es el tiempo en el cual todas las actividades se han terminado.

Una heurística es una regla que indica a qué actividad darle prioridad cuando la limitación de recursos obliga a seleccionar entre dos o más actividades disponibles. Ésta se puede aplicar en cualquier momento de la aplicación del procedimiento, y a menudo cada vez que se termina una actividad (se liberan recursos y se vuelven candidatas algunas actividades). En particular, cuando se aplica una heurística, típicamente existen actividades terminadas, algunas que no se han iniciado, y otras en proceso, y la regla heurística puede basarse en conceptos como los siguientes:

- El tiempo de iniciación temprana de una actividad que no se ha iniciado es el menor tiempo al que puede iniciarse la actividad, considerando sólo las relaciones de precedencia (pero no las restricciones de recursos).

- La holgura de una actividad que no se ha iniciado es el tiempo que puede ser retrasado el tiempo de iniciación temprana de una actividad sin afectar el tiempo de terminación del proyecto (sin considerar restricciones de recursos).

- Actividades críticas son aquellas actividades que no se han iniciado, y que tienen holgura igual a cero.
- Los seguidores de una actividad son las actividades que requieren que la actividad esté terminada para poder ser ejecutadas.

- La duración de una actividad es el tiempo total que requiere la ejecución de la misma.

\section{Heurísticas Implantadas}

En el sistema desarrollado se implantaron tres reglas heurísticas bien conocidas en la administración de proyectos:

- La regla de menor duración (MD) es la que da prioridad a la actividad con menor duración (véase, por ejemplo [8]).

- La regla de mayor número de seguidores críticos (MNSC) es la que da prioridad a la actividad con mayor número de seguidores con holgura igual a cero (véase, por ejemplo [5]).

- La regla de menor holgura (MH) es la que da prioridad a la actividad con menor holgura véase, por ejemplo [5]).

Se han hecho estudios empíricos en los cuales se comparan los tiempos de ciclo obtenidos con cada una de las tres heurísticas mencionadas, y éstos a su vez con los tiempos de ciclo óptimos. La que por lo general difiere menos del tiempo óptimo es la heurística $\mathrm{MH}$ [3].

Con el objetivo de comparar el desempeño de estas tres heurísticas en casos derivados de las órdenes de calentadores, se programaron las tres heurísticas en Visual Basic y se probó el sistema con cada una de ellas para cinco órdenes de calentadores. En todos los casos el tiempo de duración menor se obtuvo con el uso de la heurística $\mathrm{MH}$; por esto se eligió trabajar inicialmente con esta heurística. Adicionalmente, se observó que al programar inmediatamente actividades candidatas que no son críticas, se podían inmovilizar recursos que posteriormente podrían ser útiles para programar actividades críticas, por lo que se consideró modificar el algoritmo que utiliza la heurística $\mathrm{MH}$, para permitir tener tiempos de ocio en los recursos, esperando a que las actividades críticas sean candidatas y se puedan ejecutar. Así se diseñó una nueva heurística cuyo procedimiento se basa en la heurística $\mathrm{MH}$, pero permitiendo tiempos de ocio en los recursos, por lo que se le denominó heurística de menor holgura con tiempos de ocio (MHTO). 
La heurística MHTO funciona de la siguiente manera: al terminar una actividad, se verifica si existen actividades no programadas que cumplen: (a) la actividad es crítica y se han ejecutado todos sus precedentes, y (b) la actividad no es candidata debido a limitaciones en los recursos. Cuando esto sucede, en ciertas ocasiones el retraso en la ejecución de una actividad no crítica puede permitir la programación de la actividad crítica, y reducir el tiempo final del proyecto. Bajo la heurística MHTO se propone un cambio en la programación de las actividades si se cumplen las condiciones siguientes:

- Que al liberar los recursos de una actividad que se está ejecutando (llamada actividad saliente), se pueda ejecutar la actividad crítica.

- Que la actividad saliente haya tenido holgura en el momento en que se programó.

- Que al recalcular la duración del proyecto (sin programar a la actividad saliente y sin considerar las restricciones de recursos), la nueva duración del proyecto no sea mayor que la anterior.

\section{Ejemplo 1}

Para ilustrar la aplicación de la heurística MHTO, a continuación se presenta un pequeño ejemplo en que se considera que en la fábrica hay cuatro máquinas, M1, M2, M3 y M4, y dos operarios para realizar todas las actividades. La red de actividades se ilustra en la Fig. 1, $\mathrm{y}$ las duraciones y requerimientos de recursos se presentan en la Tabla 4.

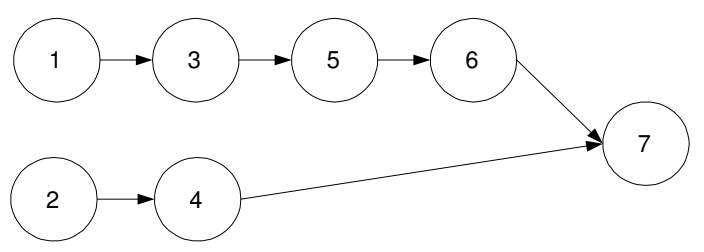

Fig. 1 Red de actividades del Ejemplo 1.

Tabla 4. Datos del Ejemplo 1.

\begin{tabular}{|c|c|c|c|}
\hline Actividad & $\begin{array}{c}\text { Duración } \\
\text { (Minutos) }\end{array}$ & Precedentes & Recursos \\
\hline 1 & 3 & & $\mathrm{M} 1, \mathrm{O}$ \\
\hline 2 & 2 & & $\mathrm{M} 1, \mathrm{O}$ \\
\hline 3 & 4 & 1 & $\mathrm{M} 2, \mathrm{O}$ \\
\hline 4 & 3 & 2 & $\mathrm{M} 3, \mathrm{O}$ \\
\hline 5 & 3 & 3 & $\mathrm{M} 3, \mathrm{O}$ \\
\hline 6 & 5 & 5 & $\mathrm{M} 4, \mathrm{O}$ \\
\hline 7 & 1 & 4,6 & $\mathrm{M} 1, \mathrm{O}$ \\
\hline
\end{tabular}

Como se ilustra en la Tabla 5, al inicio $(t=0)$ se tienen dos actividades candidatas: la 1 y la 2 , como la actividad 1 es crítica (no tiene holgura) se elige ésta para ser ejecutada, y la actividad 2 no se programa por falta de recursos. Al terminar la actividad $1(t=3)$, un operario comienza la actividad 3 , y el otro operario comienza la 2. Cuando finaliza la actividad $2(t=5)$, la única actividad disponible es la 4 por lo que ésta se elige para ser ejecutada. Posteriormente, cuando la actividad 3 termina $(t=7)$ no puede empezar a ejecutarse la actividad 5, ya que la máquina está siendo utilizada. En este momento, se considera hacer un cambio en la programación de actividades. Se determina si se cumplen los tres requisitos para el cambio:

- Al liberar los recursos de la actividad 4, si puede empezar a ejecutarse la actividad 5 .

- La actividad 4 si tenía holgura al ser elegida.

- La duración total del proyecto con el cambio de programación de actividades es menor que el tiempo que se tenía estimado sin él.

Como se cumplen las tres condiciones, se hace el cambio en la programación de actividades. Se pospone la ejecución de la actividad 4 (s.4) y en $t=7$ la actividad 5 empieza a ser ejecutada por cualquiera de los operarios (p.5). Más adelante, cuando la actividad 5 finaliza en $t=10$, se tienen dos operarios libres y dos actividades disponibles, la 4 y la 6 , y como requieren de distintas máquinas, se seleccionan las dos actividades. En $t=13$, al terminar la actividad 4, no hay actividades disponibles así que es hasta $t=15$ cuando los precedentes de la actividad 7 están terminados y se comienza esta actividad. El tiempo de ciclo en este caso es de 16 minutos, siendo conveniente mencionar que en este ejemplo, la aplicación de las heurísticas MD, MNSC y MH proporcionan tiempos de ciclo de 18, 17, y 17 minutos, respectivamente.

Tabla 5. Aplicación de la heurística MHTO.

\begin{tabular}{|c|c|c|c|c|c|c|c|}
\hline Tiempo $(\boldsymbol{t})$ & 0 & 3 & 5 & 7 & 10 & 15 & 16 \\
\hline $\begin{array}{c}\text { Actividades } \\
\text { candidatas }\end{array}$ & 1,2 & 2,3 & 4 & 5 & 4,6 & 7 & - \\
\hline $\begin{array}{c}\text { Actividades } \\
\text { programadas }\end{array}$ & 1 & 2,3 & 4 & p. 5 & 4,6 & 7 & \\
\hline
\end{tabular}

\section{Aplicación al Caso de la Producción de Calentadores}

A continuación se presentan los datos necesarios y los resultados obtenidos en la aplicación de las heurísticas 
MH y MHTO al caso de la producción de un calentador del tipo CE- 15 a 80 lt. En la Tabla 6 se muestran los datos de las actividades que se requieren en la producción de un calentador eléctrico de 15 a 80 litros.

Tabla 6. Datos del grupo CE-15 a 80 litros.

\begin{tabular}{|c|c|c|c|}
\hline Act & Duración & Precedentes & Recursos \\
\hline 1 & 14,1 & & M5, P \\
\hline 2 & 14 & 1 & $\mathrm{M} 27$ y $M 35, \mathrm{P}$ \\
\hline 3 & 5,2 & 2 & $\mathrm{M} 10, \mathrm{P}$ \\
\hline 4 & 3,9 & 3 & M31 o M32, $P$ \\
\hline 5 & 15,5 & & M5, P \\
\hline 6 & 7,3 & 5 & M15 o M16, P \\
\hline 7 & 4,5 & 5 & M15 o M16, P \\
\hline 8 & 16,2 & 6,7 & $\mathrm{M} 1, \mathrm{P}$ \\
\hline 9 & 39,2 & 8 & $\mathrm{M} 22, \mathrm{P}$ \\
\hline 10 & 8,4 & 9 & M31 o M32, $\mathrm{P}$ \\
\hline 11 & 4,4 & 10 & M31 o M32, $\mathrm{P}$ \\
\hline 12 & 2,9 & 9 & M31 o M32, $\mathrm{P}$ \\
\hline 13 & 31,65 & $4,11,12$ & M31 o M32, $P$ \\
\hline 14 & 3,9 & 13 & M31 o M32, $P$ \\
\hline 15 & 3,55 & 14 & M31 o M32, $\mathrm{P}$ \\
\hline 16 & 5,85 & 13 & M31 o M32, $\mathrm{P}$ \\
\hline 17 & 21,5 & 15,16 & $\mathrm{P}$ \\
\hline 18 & 11,3 & 17 & $\mathrm{P}$ \\
\hline 19 & 2 & 18 & $\mathrm{P}$ \\
\hline 20 & 960 & 19 & \\
\hline 21 & 9,75 & 20 & $\mathrm{P}$ \\
\hline 22 & 1,4 & 21 & $\mathrm{P}$ \\
\hline 23 & 6,4 & 22 & $\mathrm{P}$ \\
\hline 24 & 1,4 & 23 & $\mathrm{P}$ \\
\hline 25 & 1,4 & 24 & $\mathrm{P}$ \\
\hline 26 & 4,4 & 25 & $\mathrm{P}$ \\
\hline 27 & 9,4 & 26 & $\mathrm{P}$ \\
\hline 28 & 16 & & M5, P \\
\hline 29 & 7,5 & 28 & M9, P \\
\hline 30 & 1,4 & 28 & $\mathrm{P}$ \\
\hline 31 & 5,9 & 30 & $\mathrm{M} 20$ o $\mathrm{M} 21, \mathrm{P}$ \\
\hline 32 & 15,3 & & M5, P \\
\hline 33 & 17,2 & 32 & $\mathrm{M} 1, \mathrm{P}$ \\
\hline 34 & 15,2 & 32 & $\mathrm{M} 1, \mathrm{P}$ \\
\hline 35 & 4,6 & 33,34 & $\mathrm{M} 3, \mathrm{P}$ \\
\hline 36 & 3,5 & 35 & $\mathrm{M} 20$ o M21, $\mathrm{P}$ \\
\hline 37 & 4,7 & 36 & $\mathrm{M} 31$ o $M 32, \mathrm{P}$ \\
\hline 38 & 22,5 & $29,31,37$ & $\mathrm{P}$ \\
\hline 39 & 18 & 38 & $\mathrm{P}$ \\
\hline 40 & 8,3 & 27,39 & $\mathrm{P}$ \\
\hline 41 & 21,2 & 40 & $\mathrm{P}$ \\
\hline 42 & 10,3 & 41 & $P$ \\
\hline 43 & 1,5 & 42 & $\mathrm{P}$ \\
\hline 44 & 5,2 & 43 & $\mathrm{P}$ \\
\hline
\end{tabular}

Revista Facultad de Ingeniería, Chile, Vol.12 N², 2004
Tabla 7. Secuenciación de actividades con MHTO.

\begin{tabular}{|c|c|c|}
\hline Tiempo & Act. Candidatas & Act. Seleccionadas \\
\hline 0 & $1,5,28,32$ & 5 \\
\hline 15,5 & $1,6,7,28,32$ & 6,7 \\
\hline 20 & $1,28,32$ & 1 \\
\hline 22,8 & 8 & $\mathrm{~s} 8, \mathrm{p} 1$ \\
\hline 22,8 & $1,28,32$ & 1 \\
\hline 36,9 & $2,28,32$ & 2 \\
\hline 39 & $9,28,32$ & 9 \\
\hline 50,9 & $3,28,32$ & 3 \\
\hline 56,1 & $4,28,32$ & 4 \\
\hline 60 & 28,32 & 32 \\
\hline 75,3 & $28,33,34$ & 33 \\
\hline 78,2 & $10,12,28$ & 10 \\
\hline 86,6 & 11 & $\mathrm{~s} 33, \mathrm{p} 11$ \\
\hline 86,6 & $12,28,33,34$ & 12 \\
\hline 89,5 & $28,33,34$ & 33 \\
\hline 91 & 13,28 & 13 \\
\hline 106,7 & 28,34 & 34 \\
\hline 121,9 & 28,35 & 28 \\
\hline 122,65 & $14,16,35$ & 14 \\
\hline 126,55 & 16 & $\mathrm{~s} 16, \mathrm{p} 28$ \\
\hline 126,55 & $15,28,35$ & 15 \\
\hline 128,5 & 28,35 & 28 \\
\hline 130,1 & 17 & $\mathrm{~s} 17, \mathrm{p} 28$ \\
\hline 130,1 & 28,35 & 28 \\
\hline 146,1 & $29,30,35$ & 35 \\
\hline 150,7 & $29,30,36$ & 36 \\
\hline 151,6 & 18 & $\mathrm{~s} 18, \mathrm{p} 36$ \\
\hline 151,6 & $29,30,36$ & 36 \\
\hline 155,1 & $29,30,37$ & 29 \\
\hline 162,6 & 30,37 & 30 \\
\hline 162,9 & 19,37 & 19 \\
\hline 164 & 31,37 & 31 \\
\hline 164,9 & 20,37 & 20,37 \\
\hline 169,9 & 38 & 38 \\
\hline 192,4 & 39 & 39 \\
\hline 1124,9 & 21 & 21 \\
\hline 1134,65 & 22 & 22 \\
\hline 1136,05 & 23 & 23 \\
\hline 1142,45 & 24 & 24 \\
\hline 1143,85 & 25 & 25 \\
\hline 1145,25 & 26 & 26 \\
\hline 1149,65 & 27 & 27 \\
\hline 1159,05 & 40 & 40 \\
\hline 1167,35 & 41 & 41 \\
\hline 1188,55 & 42 & 42 \\
\hline 1198,85 & 43 & 43 \\
\hline 1200,35 & 44 & 44 \\
\hline 1205,55 & - & FIN \\
\hline
\end{tabular}


En una corrida con los datos de producción de un CE-15 a 80 litros, la heurística de menor holgura obtuvo un tiempo de ciclo de 1208.1 y al aplicar la heurística de menor holgura con tiempos de ocio el tiempo de ciclo se redujo a 1205.55, en la Tabla 7 se muestra la secuenciación de actividades obtenida.

Es necesario mencionar que la heurística MHTO en ciertos casos, puede comportarse peor que la heurística $\mathrm{MH}$, aumentando el tiempo de ciclo del proyecto. Considerando que debido a que se aplican reglas heurísticas, el sistema desarrollado no necesariamente debe proporcionar el tiempo de ciclo óptimo, la implantación final del sistema consistió en aplicar las dos heurísticas que mejor se comportaron: $\mathrm{MH}$ y MHTO, y sugerir la programación con el menor tiempo de ciclo. De acuerdo con los resultados experimentales que a continuación se discuten, no se pudo decidir que alguna de estas heurísticas se comporte mejor que la otra.

\section{RESULTADOS EXPERIMENTALES Y CONCLUSIONES}

Con el objetivo de evaluar la conveniencia de considerar la heurística MHTO, se decidió comparar el desempeño de las heurísticas MH y MHTO en el sistema, para lo cual se generaron órdenes de producción y se aplicaron las diferentes heurísticas a cada caso generado. Los resultados indicaron que en $54 \%$ de los casos, la heurística MHTO disminuyó (mejoró) el tiempo de ciclo de la heurística $\mathrm{MH}$, mientras que en $38 \%$ de los casos lo aumentó (empeoró), en el resto de los casos (8\%) el resultado fue el mismo. En la Tabla 8 se presentan estadísticas más detalladas de los casos de disminución o aumento; por ejemplo, en los casos en que la heurística MHTO disminuyó el tiempo de ciclo de la heurística $\mathrm{MH}$, el promedio de disminución fue del $2.09 \%$, la disminución mínima fue de $0.12 \%$, y la máxima del $25.56 \%$, lo que muestra que sí conviene considerar la heurística MHTO como alternativa.

Tabla 8. Comparación de Tiempos de Ciclo de la heurística MHTO contra MH.

\begin{tabular}{|c|c|c|}
\cline { 2 - 3 } \multicolumn{1}{c|}{} & Disminución & Aumento \\
\hline Mínimo & $0.12 \%$ & $0.23 \%$ \\
\hline Promedio & $2.09 \%$ & $3.65 \%$ \\
\hline Máximo & $25.56 \%$ & $9.97 \%$ \\
\hline
\end{tabular}

Tabla 9. Comparación de Tiempos de Ciclo con diferentes tipos de órdenes.

\begin{tabular}{|l|c|c|c|}
\cline { 2 - 4 } \multicolumn{1}{c|}{} & $\begin{array}{c}\text { MHTO } \\
\text { Menor tiempo } \\
\text { de ciclo }\end{array}$ & $\begin{array}{c}\text { MH Menor } \\
\text { tiempo de } \\
\text { ciclo }\end{array}$ & Igual \\
\hline Un solo grupo & $97 \%$ & $0 \%$ & $3 \%$ \\
\hline Varios grupos & $0 \%$ & $96 \%$ & $4 \%$ \\
\hline
\end{tabular}

Como se menciona anteriormente, en la mayoría de los casos considerados la heurística MHTO mejoró a MH, aunque también se desempeñó peor que MH en otros casos. Se observó que cuando MH se comporta mejor, las órdenes estaban compuestas de varios tipos de calentadores (ver Tabla 9) y no era conveniente tener tiempos de ocio para apurar la producción de un tipo de calentador, sino que para terminar la orden en menor tiempo, era conveniente retrazar el tiempo de ciclo de algún tipo de calentador. En cambio, en las órdenes de producción donde había sólo un tipo de calentador, siempre la heurística MHTO se comportó mejor, comprobando que era mejor producir cada calentador lo más rápido posible.

\section{REFERENCIAS}

[1] R. Anupindi, S. Chopra, S.D Deshmukh, J.A. Van Mieghem y E. Zemel; Managing Business Process Flows. Prentice-Hall, Inc., New Jersey, 1999.

[2] A.L. Castillo; "Desarrollo de un Sistema para Programar Recursos y Operaciones en la Fabricación de Calentadores Eléctricos Bajo Pedido", Tesis para optar el título de Ingeniera Industrial, División de Ingenierías, Instituto Tecnológico Autónomo de México, México, 2002.

[3] E.W. Davis y J.H. Patterson; "A Comparison of Heuristic and Optimum Solutions in ResourceConstrained Project Scheduling". Management Science, 21(8), pp. 944-955, 1975.

[4] L.J. Krajewski, y L.P Ritzman; "Operations Management Strategy and Analisis". AddisonWesley Publishing Company, Inc., Massachusetts, 1996.

[5] J.R. Meredith y S.J. Mantel; Project Management: a Managerial Approach, John Wiley \& Sons Inc., New York, 2000. 
[6] A. Mingozzi, V. Maniezzo, S. Ricciardelli y L. Bianco; "An Exact Algorithm for the ResourceConstrained Project Scheduling Problem Based on a New Mathematical Formulation". Management Science, 44, 5, pp. 714-729, 1998.
[7] J.P. Stinson, E.W. Davis y B.M. Khumawala; 'Multiple Resource-Constrained Scheduling Using Branch and Bound". IIE Transactions, 10, 3, pp. 252-259, 1978.

[8] D.R. Sule; 'Industrial Scheduling". PWS Publishing Co., Boston, 1997. 